\title{
Simulation on the Thermal Properties of Polymer Composites Reinforced by Buckypaper
}

\author{
AYing Zhang ${ }^{1, a}$, ZhengHong $\mathrm{Li}^{2}$ \\ ${ }^{1}$ Harbin University, 150086 Harbin, China \\ ${ }^{2}$ Harbin Institute of Technology, 150001 Harbin, China
}

\begin{abstract}
FLUENT was used to analyze how the bending cycles of buckypaper affects the temperature distribution, the changes of the temperature with time and the heating rate during heating. The typical temperature decreases with the increase of bending cycles of the buckypaper. The typical temperature increases with the increase of heating power. The temperature distribution of the polymer composites is more uniform with the increase of bending cycles of buckypaper. The difference of the maximum and minimum temperature of the composites increases linearly with the increase of heating power. The average temperature of the composites when reaching the stable state increase as the bending cycles of buckypaper decrease. The difference of the maximum and minimum temperature of the composites decreases with the increase of the bending cycles.
\end{abstract}

\section{Introduction}

CNTs have been regarded as one of the most promising reinforcement materials or functional agents for developing high performance structural and multifunctional nanocomposites [1-3]. A new approach was developed by the authors to infiltrate a preformed nanotube network or nanotube mat (often called buckypaper) with resin to produce bulk polymeric nanocomposites with a uniform CNT dispersion, wellcontrolled nanostructures and high CNT loading capability [4]. SWCNT-BPs can be used to fabricate high-loading CNT/thermoplastic composites with improved thermal properties [5]. With their excellent thermal conductivity, the polymer composites reinforced by the buckypaper can be used to solve the heat problems associated with advanced electronic equipment, radiators, connectors and printed circuit boards. With the effective control of nanopaper of carbon nanotubes, they can be used as good sensor and functional material. Studies on their thermal properties are less frequently reported, particularly for the thermal properties of buckypaper reinforced polymer composites analyzed by using the finite element analysis (FEA). In this study, heating model of the polymer composite reinforced by buckypaper is established to predict the thermal property of buckypaper reinforced composite.

\section{Finite element models}

As shown in Figure 1, the heating model of the polymer composites reinforced by the bent buckypaper under different heating conditions were developed by using the finite element program FLUENT. Effects of the bending cycles of the buckypaper on the temperature distribution and heating uniformity of the polymer composites reinforced by the bent buckypaper were investigated using finite element software FLUENT.

In Figure 1, heating the buckypaper is driven by a power source, and the cube region is the polymer matrix which is heated by the buckypaper during heating process. The temperature of the polymer matrix is increased due to the electro-heating of the buckypaper. The length, width, and the thickness of the heating model of the polymer composite reinforced by the bent buckypaper are $36 \mathrm{~mm}, 5 \mathrm{~mm}$, and $10 \mathrm{~mm}$ respectively. The thicknesses of the buckypaper are $0.4 \mathrm{~mm}$. The bending height and bending period (A) of the bent buckypaper are $6 \mathrm{~mm}$ and $12 \mathrm{~mm}$. The objective of this research is to analyze the effect of the bending cycles of the buckypaper on the thermal conductivity of the composites using a finite element software FLUENT.

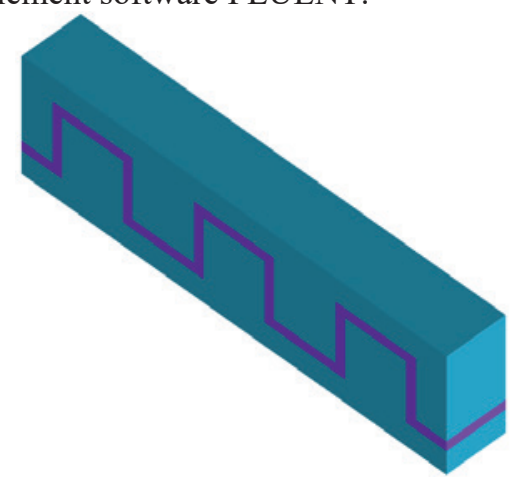

$\underset{\text { Riso }}{\text { ANSYS }}$

Figure 1. Model of the polymer composites reinforced by bent buckypaper

\footnotetext{
${ }^{\mathrm{a}}$ Corresponding author: zaying@sina.com
} 
The grid work is done by software ICEM, and the mesh model of the polymer composite reinforced by the bent buckypapers as shown in Figures 2.

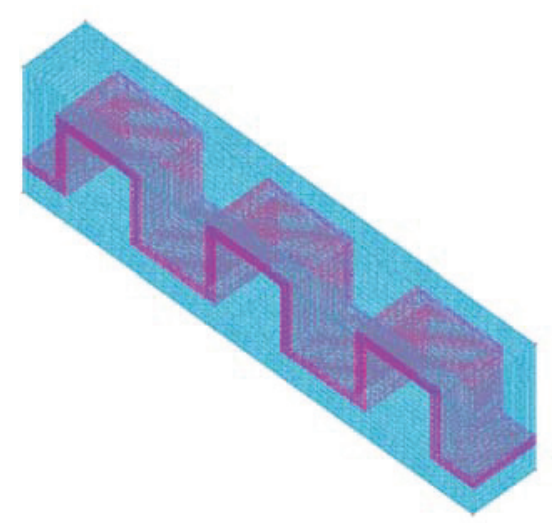

ANSYS

Figure 2. Mesh division model of the polymer composites reinforced by the bent buckypaper

\section{Results and discussion}

As shown in Table 1, the maximum, minimum and average temperature of the composites reinforced by the bent buckypaper with different bending cycles under different power along the section $\mathrm{z}=0$ were calculated by the finite element software FLUENT.

Table 1. Typical temperature of composites reinforced by the bent buckypaper with different bending cycles under different power along the section $\mathrm{z}=0$

\begin{tabular}{|c|c|c|c|c|c|c|}
\hline \multirow{2}{*}{$\begin{array}{c}\text { Bending } \\
\text { cycles }\end{array}$} & \multicolumn{3}{|c|}{$\mathbf{0 . 3 w}$} & \multicolumn{3}{c|}{$\mathbf{0 . 6 w}$} \\
\cline { 2 - 7 } & $\begin{array}{c}\text { Tmax } \\
/ \mathbf{K}\end{array}$ & $\begin{array}{c}\text { Tmin } \\
/ \mathbf{K}\end{array}$ & $\begin{array}{c}\text { Tave } \\
/ \mathbf{K}\end{array}$ & $\begin{array}{c}\text { Tmax } \\
/ \mathbf{K}\end{array}$ & $\begin{array}{c}\text { Tmin } \\
/ \mathbf{K}\end{array}$ & $\begin{array}{c}\text { Tave } \\
/ \mathbf{K}\end{array}$ \\
\hline 1 & 377.8 & 354.6 & 370.7 & 455.7 & 409.3 & 441.5 \\
\hline 3 & 330.9 & 319.9 & 327.6 & 361.7 & 339.9 & 355.1 \\
\hline 5 & 319.1 & 312.0 & 317.1 & 338.1 & 324.0 & 334.2 \\
\hline
\end{tabular}

Table 1 shows that the maximum, minimum and average temperature decrease as the bending cycles of the buckypaper increase from 1 A to 3 A under the same power. As shown in Table 1, the maximum, minimum and average temperature increases as the heating power increase from $0.3 \mathrm{~W}$ to $0.6 \mathrm{~W}$ with the same bending cycle.

Figure 3 shows the temperature cloudy maps which indicate that the temperature distribution of the polymer composites reinforced by the bent buckypaper along the section $\mathrm{z}=0$ is more uniform as the bending cycles of the buckypaper increase from $1 \mathrm{~A}$ to $3 \mathrm{~A}$ under the heating power of $0.3 \mathrm{~W}$.

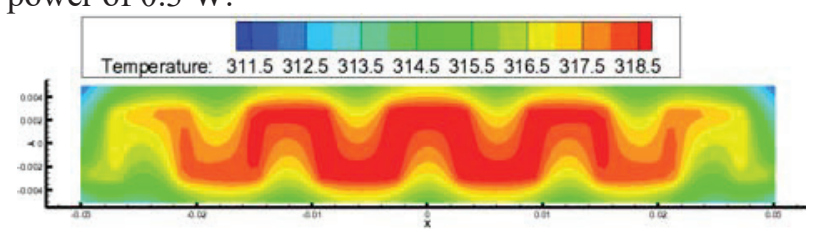

(a) $5 \mathrm{~A}$

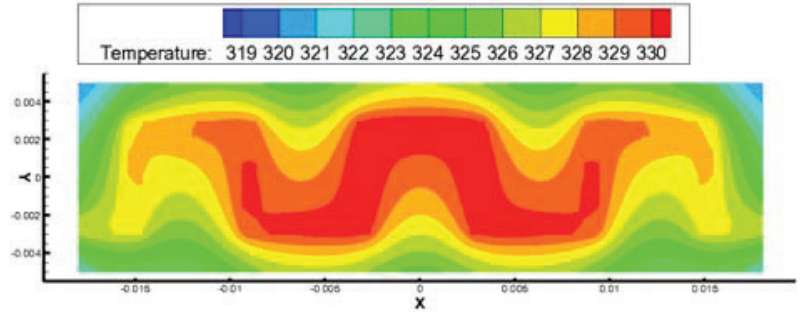

(b) $3 \mathrm{~A}$

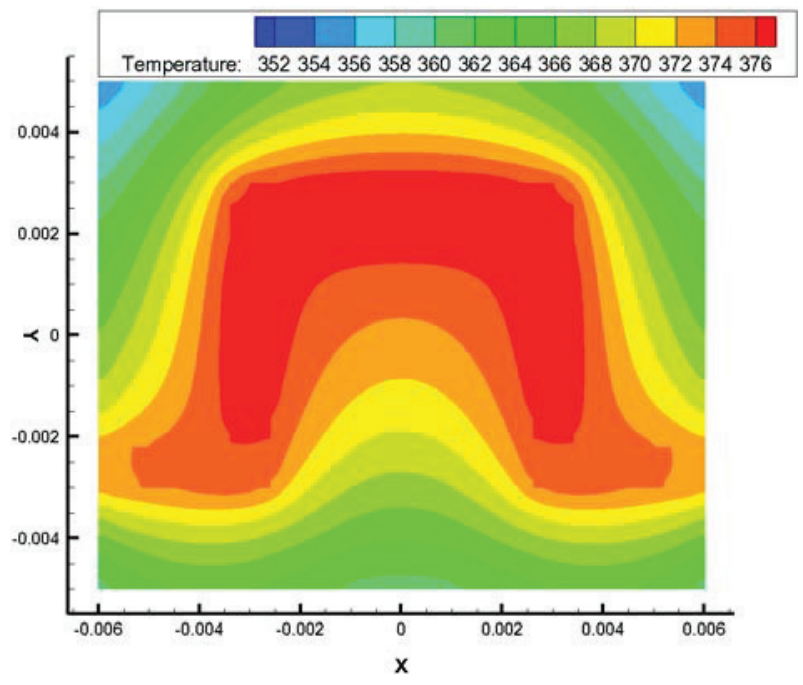

(c) $1 \mathrm{~A}$

Figure 3. Temperature distribution of nanocomposite with different bending cycles along the section $\mathrm{z}=0$ under the heating power of $0.3 \mathrm{w}$

Figure 4 shows the temperature cloudy maps which indicate that the temperature distribution of the polymer composites reinforced by the bent buckypaper along the section $\mathrm{z}=0$ is more uniform as the bending cycles of the buckypaper increase from $1 \mathrm{~A}$ to $3 \mathrm{~A}$ under the power of $0.6 \mathrm{~W}$.

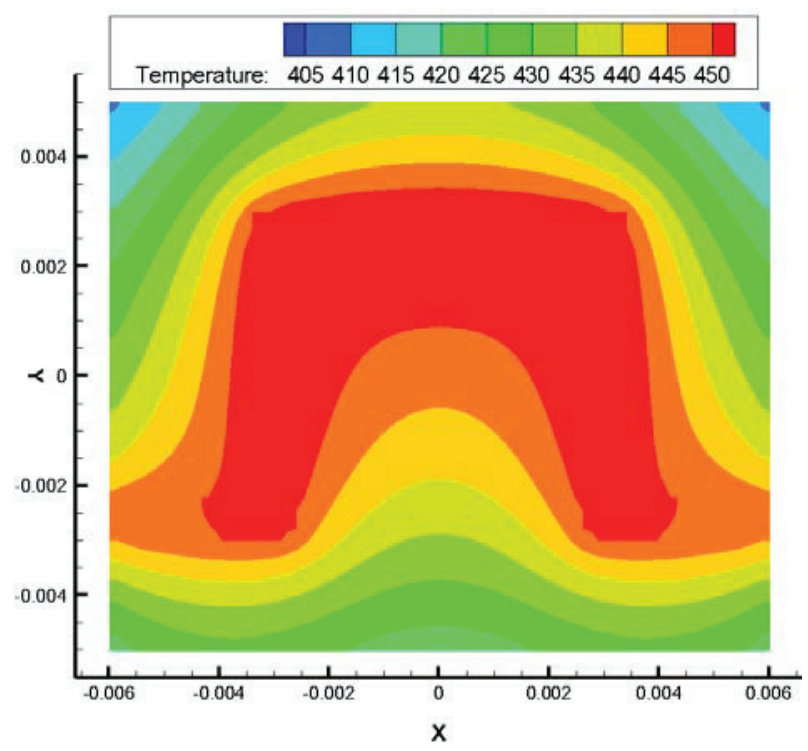

(a) $1 \mathrm{~A}$ 


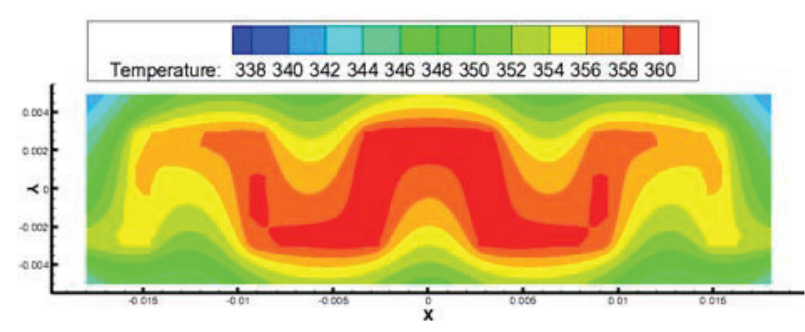

(b) $3 \mathrm{~A}$

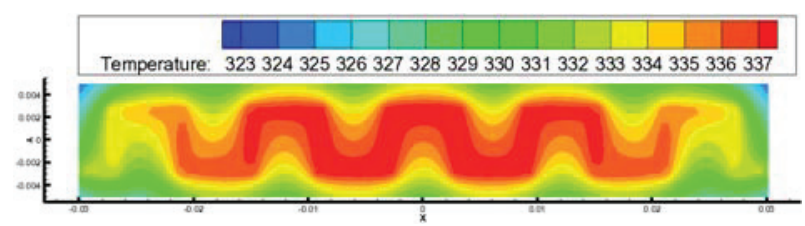

(c) $5 \mathrm{~A}$

Figure 4. Temperature distribution of composites reinforced by buckypaper with different bending cycles along the section $\mathrm{z}=0$ under the heating power of $0.6 \mathrm{w}$

Figure 3 and 4 shows the temperature cloudy maps which indicate that the temperature distribution of the polymer composites reinforced by the bent buckypaper along the section $\mathrm{z}=0$ is more uniform as the bending cycles of the buckypaper increase from $1 \mathrm{~A}$ to $5 \mathrm{~A}$.

Table 2 shows that the average thermal flow of composites reinforced by buckypaper with different bending cycles under the heating power of $0.3 \mathrm{~W}$ and 0.6 $\mathrm{W}$ along external surface. As shown in Table 2, the average thermal flow of both the buckypaper and resin decrease as the bending cycles of buckypaper increase with the same heating power.

Table 2. Average thermal flow of composites reinforced by buckypaper with different bending cycles under the heating power of $0.3 \mathrm{~W}$ and $0.6 \mathrm{~W}$ along external surface $\left(\mathrm{W} / \mathrm{m}^{2}\right)$

\begin{tabular}{|c|c|c|c|c|}
\hline \multirow{2}{*}{$\begin{array}{c}\text { Bending } \\
\text { cycles }\end{array}$} & \multicolumn{2}{|c|}{ 0.3w } & \multicolumn{2}{c|}{ 0.6w } \\
\cline { 2 - 5 } & Buckypaper & Resin & Buckypaper & Resin \\
\hline 1 & 748.13 & 641.86 & 1496.65 & 1284.06 \\
\hline 3 & 287.88 & 250.70 & 501.08 & 575.38 \\
\hline 5 & 178.10 & 155.77 & 356.33 & 311.68 \\
\hline
\end{tabular}

Figure 5 shows the curve of temperature difference of composites reinforced by buckypaper with different bending cycles along the section $\mathrm{z}=0$. As shown in Figure 5 , the difference of the maximum and minimum temperature of the composite increases linearly with the increase of the heating power. Figure 5 shows that the difference of the maximum and minimum temperature of the composite decreases with the increase of the bending cycles of buckypaper, which indicate that the temperature distribution of the composite is more uniform.

Figure 6 shows the curve of average temperature of composites reinforced by buckypaper with different bending cycle versus time along the section $\mathrm{z}=0$. Figure 6 shows that both curves of the average temperature exhibit similar trend and there are two distinct stages observed.
The first stage shows that the slopes of the curves increase significantly with increasing the heating time. A fast increase of the average temperature occurs during stage I after which the slope is changed, indicating the beginning of the second stage. A progressive but slow increase of the average temperature is observed in the second stage.

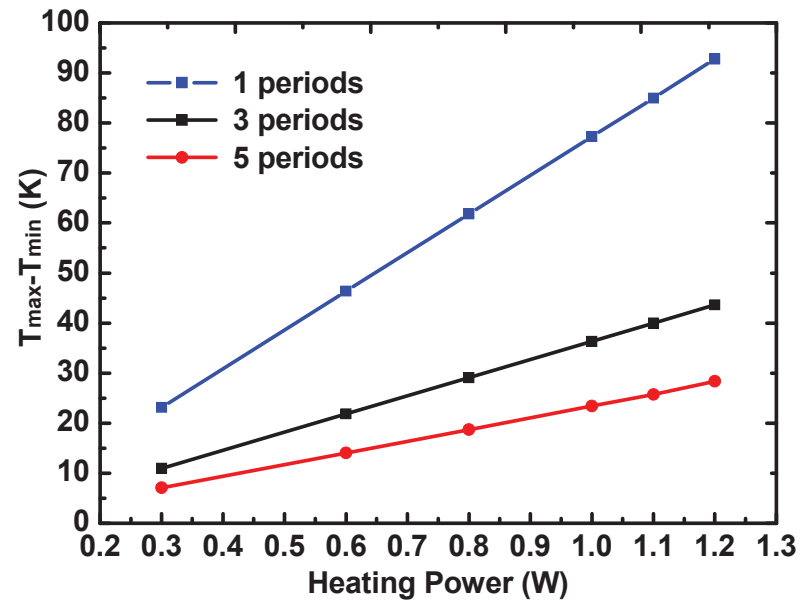

Figure 5. Curve of temperature difference of composites reinforced by buckypaper with different bending cycles along the section $\mathrm{z}=0$

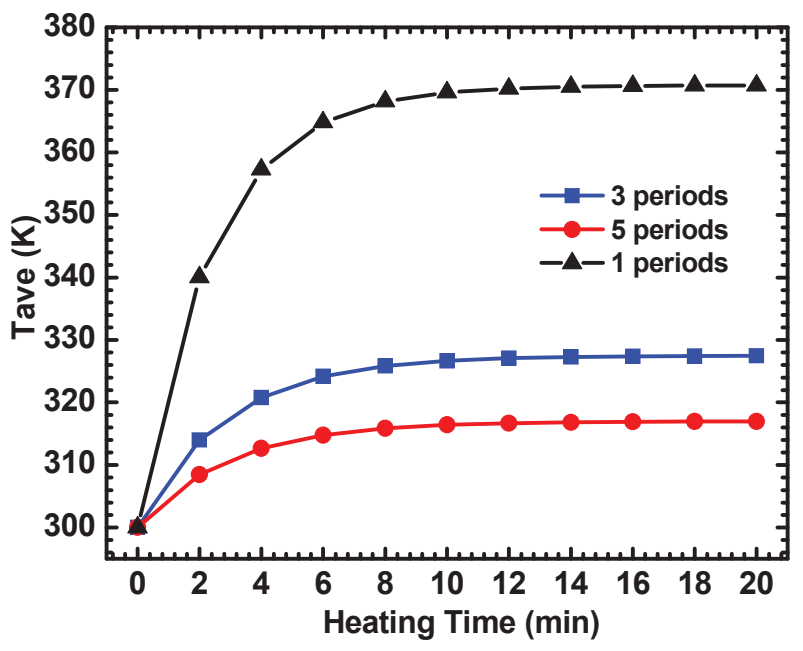

Figure 6. Curve of average temperature of composites reinforced by buckypaper with different bending cycle versus time along the section $\mathrm{z}=0$

As shown in Figure 6, the heating time reaching the stable state is about 10 minutes for the polymer composites reinforced by the bent buckypaper of 5 bending cycles. The heating time reaching the stable state is about 14 minutes for the polymer composites reinforced by the bent buckypaper of 3 bending cycles. It takes longer heating time for the composites reinforced by the bent buckypaper of 1 bending cycles to reach the stable state than that of 3 and 5 bending cycles. As shown in Figure 6, the average temperature of the composites when reaching the stable state increase as the bending cycles of buckypaper decrease.

Figure 7 and 8 shows the curve of maximum and minimum temperature of composites reinforced with the bent buckypaper of different bending cycles versus time along the section $\mathrm{z}=0$. 


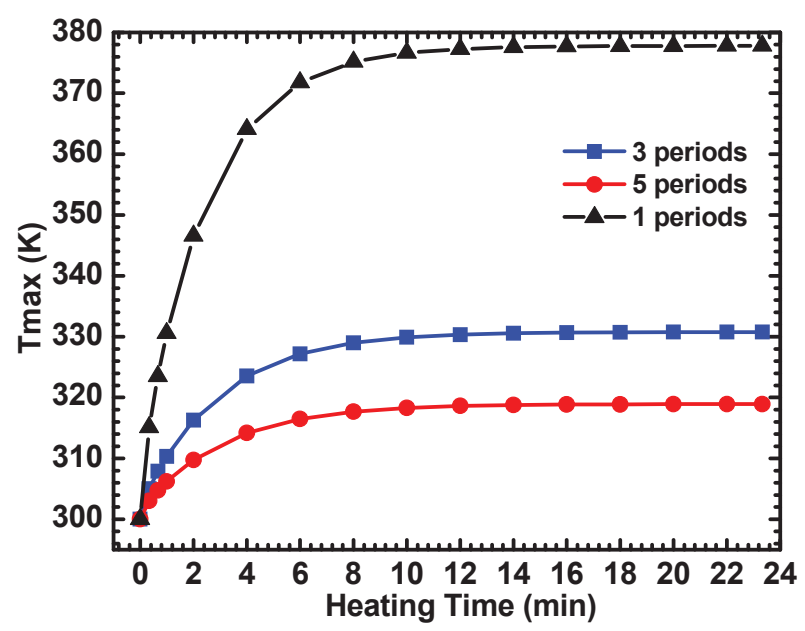

Figure 7. Curve of maximum temperature of composites reinforced with the bent buckypaper of different bending cycles versus time along the section $\mathrm{z}=0$

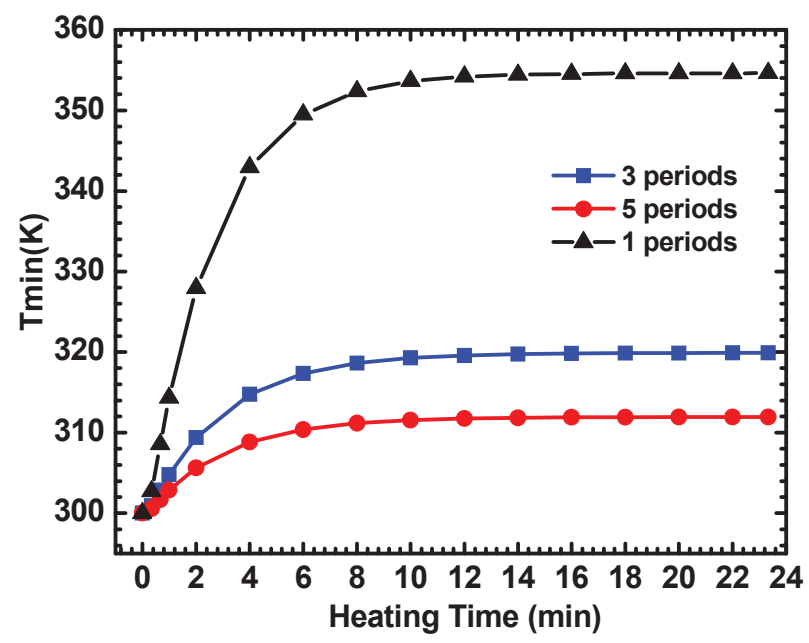

Figure 8. Curve of minimum temperature of composites reinforced with the bent buckypaper of different bending cycles versus time along the section $\mathrm{z}=0$

As shown in Figure 6 to Figure 8, the maximum, minimum and average temperature of composites reinforced with the bent buckypaper of 1 bending cycles is the most highest than that of 3 and 5 bending cycles.

Table 3. Typical temperature of composites reinforced with the bent buckypaper of 1 and 3 bending cycles versus time along the section $\mathrm{z}=0$

\begin{tabular}{|c|c|c|c|c|c|c|}
\hline \multirow{2}{*}{$\begin{array}{c}\text { Time } \\
/ \mathbf{s}\end{array}$} & \multicolumn{3}{|c|}{1} & \multicolumn{3}{c|}{3} \\
\cline { 2 - 7 } & $\begin{array}{c}\mathbf{T}_{\max } \\
/ \mathbf{K}\end{array}$ & $\begin{array}{c}\mathbf{T}_{\min } \\
/ \mathbf{K}\end{array}$ & $\begin{array}{c}\mathbf{T}_{\text {ave }} \\
/ \mathbf{K}\end{array}$ & $\begin{array}{c}\mathbf{T}_{\max } \\
/ \mathbf{K}\end{array}$ & $\begin{array}{c}\mathbf{T}_{\min } \\
/ \mathbf{K}\end{array}$ & $\begin{array}{c}\mathbf{T}_{\text {ave }} \\
/ \mathbf{K}\end{array}$ \\
\hline 0 & 300 & 300 & 300 & 300 & 300 & 300 \\
\hline 120 & 346.6 & 328.0 & 340.0 & 316.3 & 309.4 & 314.0 \\
\hline 480 & 375.2 & 352.4 & 368.1 & 329.0 & 318.6 & 325.8 \\
\hline 960 & 377.7 & 354.5 & 370.6 & 330.7 & 319.8 & 327.4 \\
\hline 1080 & 377.8 & 354.6 & 370.7 & 330.7 & 319.9 & 327.4 \\
\hline 1440 & 377.8 & 354.6 & 370.7 & 330.8 & 319.9 & 327.5 \\
\hline
\end{tabular}

Table 3 shows the typical temperature of composites reinforced with the bent buckypaper of 1 and 3 bending cycles versus time along the section $\mathrm{z}=0$. As shown in Table 3 and 4 , the difference of the maximum and minimum temperature of the composite decrease with the increase of the bending cycles, which indicate that the temperature distribution of the composite is more uniform.

\section{Conclusions}

FLUENT was used to analyze how the bending cycles of buckypaper affects the temperature distribution, the changes of the temperature with time and the heating rate during heating.

The maximum, minimum and average temperature decreases as the bending cycles of the buckypaper increase from $1 \mathrm{~A}$ to $3 \mathrm{~A}$ under the same power. The maximum, minimum and average temperature increases as the heating power increase from $0.3 \mathrm{~W}$ to $0.6 \mathrm{~W}$ with the same bending cycle. The temperature distribution of the polymer composites reinforced by the bent buckypaper along the section $\mathrm{z}=0$ is more uniform with the increase of bending cycles of buckypaper. The average thermal flow of both the buckypaper and resin decrease as the bending cycles of buckypaper increase with the same heating power.

The difference of the maximum and minimum temperature of the composites increases linearly with the increase of the heating power. The difference of the maximum and minimum temperature of the composite decreases with the increase of the bending cycles of buckypaper, which indicate that the temperature distribution of the composite is more uniform.

The average temperature of the composites when reaching the stable state increase as the bending cycles of buckypaper decrease. The difference of the maximum and minimum temperature of the composites decrease with the increase of the bending cycles, which indicate that the temperature distribution of the composite is more uniform.

\section{Acknowledgement}

This work was financially supported by Heilongjiang Postdoctoral Scientific Research Developmental Fund (Grant No. LBH-Q16141).

\section{References}

1. R. S. Ruoff, D. C. Lorents. Carbon. 33, 7(1995)

2. E. T. Thostenson, Z. F. Ren, T. W. Chou. Compos Sci Technol, 61, 13(2001)

3. K.T. Lau, D. Hui. Compos Part B-Eng, 33, 4(2002)

4. Z. Liang, J. Gou, C. Zhang, et al. Mater Sci Eng A, 365, 1-2(2004)

5. A. M. Díez-Pascual, J. W. Guan, B. Simard, et al. Compos Part A-Appl S, 43, 6(2012) 